\title{
5 Creating Illusion Effects Using Transformation Optics
}

\author{
Yun Lai ${ }^{1}$, Jack $\mathrm{Ng}^{2}$ and C.T. Chan ${ }^{2 *}$ \\ ${ }^{1}$ School of Physical Science and Technology, Soochow University, Suzhou, China \\ ${ }^{2}$ Department of Physics, Hong Kong University of Science and Technology, Hong Kong
}

Abstract:

In this chapter, we show how to create illusion effects using metamaterials. We will see that a passive metamaterial device can be designed such that when it is placed next to or covering an object, the scattered fields of the object and the device together will changed to be exactly the same as the scattered fields due to another object. Simply put, we can turn an object optically and stereoscopically into another one. An apple can be made to look like a banana. If we make a measurement of the electromagnetic fields at the designed frequency, there is no way to distinguish optically between the true object and the illusion. Invisibility can be regarded as a special case of the illusion effect, in which case the object is turned optically to a volume of free space. The metamaterial does not need to encircle the object to create the illusion effect. If we use this method to implement invisibility, the cloaked object will not be blinded by the cloak.

The design is based on the replacement of optical spaces and the material parameters are determined using the technique of transformation optics. One route to achieve illusion effects is to employ the idea of "complementary media". The materials designed using "complementary media" typically contain negative refractive index components and no extreme values of constitutive parameters are needed. A slight variation of the scheme can create a variety of effects. For example, we can make an object appear larger in size, appear rotated or located at other positions. Illusion optics may lead to some plausible applications, such as small or reduced form-factor optical devices that exhibit the same optical functions as much larger instruments, and or we can create a "super-absorber" that can absorb significantly more than its geometric cross section.

\section{Metamaterials}

In this chapter, we will see that negative index metamaterials can create illusion effects. In the 1960s, Veselago proposed that some exotic optical phenomena like 
negative refraction may occur in "abnormal" materials with simultaneously negative permittivity and permeability [1]. While negative permittivity can be found in natural materials, negative permeability is much more difficult to obtain. It is even more difficult for both to be negative at the same frequency. Negative refraction hence remains largely an academic curiosity until artificial magnetism was proposed [2] and realized experimentally [3]. To date, advances in research on metamaterials have paved the way for realizing new materials with nearly arbitrary values of permittivity and permeability tensor components. Even position dependent, anisotropic, tunable and reconfigurable material parameters can be designed and made. Such advances enable us to realize not only negative refractive index and zero refractive index, but more exotic and intriguing effects such as invisibility and illusion.

Metamaterials are typically composed of an array of man-made subwavelength metallic resonators embedded in a dielectric medium. For such an inhomogenous media, the components of permittivity and permeability tensors are effective material parameters obtained conceptually and operationally through the process of homogenization. As one of the key component of the metamaterial needed to realize "illusion optics" is negative refractive index, we show in Fig. 1 the most straightforward method to obtain a negative refractive index, as first proposed by Pendry et al. [1] and Smith et al. [4] In this design, artificial permeability is achieved using a double split ring resonator structure, in which the oscillating electric currents with opposite phases in the two split rings provide a controllable response to the magnetic field in the $z$ direction, as shown in Fig. 1(a). The resonant response to the time varying change of magnetic flux results in a susceptibility that can be out of phase to the driving field, giving the possibility of a negative response. If the response of the individual resonator is strong enough and if the density of such resonators inside the embedding medium is sufficiently high, we can obtain negative permeability. Negative permittivity is obtained using metallic wires of a finite length that have resonant response to external electric field. If the system parameters are designed such that a negative response to both of the electric and magnetic fields occurs at the same frequency range, a pass band with negative group velocity is obtained and as long as the resonators are subwavelength, the metamaterials can be described by effective negative permittivity and permeability. 
(a)

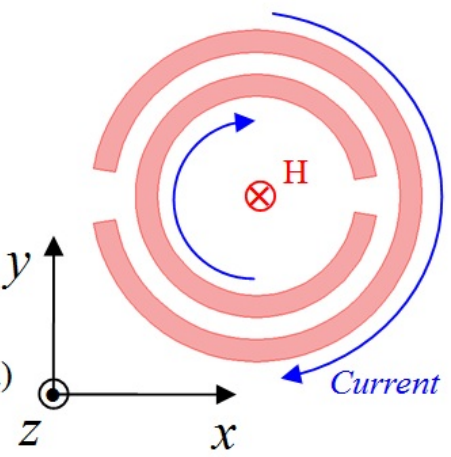

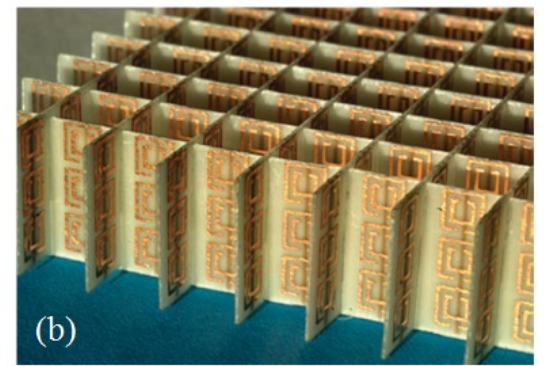

Fig. 1. (a) Schematic illustration of the double split ring resonator that has a controllable response to magnetic field in the $z$ direction. (b) A double negative metamaterial composed of split ring resonators and wires that was used to experimentally demonstrate negative refraction [quote original reference].

It is difficult to scale the structures realized by Smith and co-workers as shown in Fig. 1 down to infra-red and optical frequencies. Absorption of the metallic components will also compromise the operation at high frequencies. But with rapid advances in nanotechnology fabrication techniques [5,6] and new ideas such as loss compensation [7], metamaterials with nearly arbitrary values of constitutive parameters and operating at high frequencies become an achievable goal. In this chapter, we will not consider how to realize such metamaterials in detail, but focus on how to use metamaterials to create arbitrary optical illusions, assuming that metamaterials of arbitrary permittivity and permeability with negligible loss at a single frequency can be achieved. In most of the simulations that we show below, metamaterials of a small loss of $10^{-4}$ has been used to ensure good illusion effects. Readers interested in the design and realization of metamaterials may refer to some important works listed in the references. We also note in the outset that the theory of illusion optics applies in 2D and 3D. All the simulations shown below are for $2 \mathrm{D}$ examples as $3 \mathrm{D}$ simulations are too demanding on computation resources. All the cloaking and illusion effects discussed below only work at a narrow band of frequencies. The bandwidth is limited by the frequency dispersion of the metamaterial.

\section{Transformation optics as a design tool}

It has probably been long recognized that some wave equations are form invariant under coordinate transformations and there is a correspondence between the transformed space geometry with the new material properties. However, such 
correspondence was previously used as a mathematical technique to solve wave equations and the physical implication of such a correspondence was not fully explored. Part of the reason that such as a correspondence was not exploited is because that the parameters of the new material after transformation often become anisotropic, inhomogeneous, and especially, exhibiting extreme values not found in natural materials. However, this difficulty is overcome by the emergence of metamaterials which paves the way for introduction of the idea of transformation optics.

The concept of transformation optics has been proposed based on the form invariance of the Helmholtz equation and Maxwell's equations under coordinate transformations [8, 9]. It has soon become one of the most useful theories in the design of metamaterial applications and enables us to achieve exotic wave manipulation effects that were previously thought to be impossible.

To see how transformation optics works, let us consider Maxwell's equations at a particular frequency $\omega$ :

$$
\nabla \times \mathbf{E}+i \omega \mathbf{\mu H}=0, \nabla \times \mathbf{H}-i \omega \mathbf{E} \mathbf{E}=0,
$$

in which $\boldsymbol{\varepsilon}(\mathbf{x})$ and $\boldsymbol{\mu}(\mathbf{x})$ are the permittivity and permeability tensors. Now if we do a coordinate transformation by applying a mapping from $\mathbf{x}$ to $\mathbf{x}^{\prime}$, i.e. $\mathbf{x}^{\prime}=\mathbf{x}^{\prime}(\mathbf{x})$, then the form of Maxwell's equations in the new coordinate $\mathbf{x}^{\prime}$ is preserved, i.e.

$$
\nabla \times \mathbf{E}^{\prime}+i \omega \boldsymbol{\mu}^{\prime} \mathbf{H}^{\prime}=0, \nabla \times \mathbf{H}^{\prime}-i \omega \mathbf{\varepsilon}^{\prime} \mathbf{E}^{\prime}=0,
$$

in which the new electromagnetic fields are expressed as

$$
\mathbf{E}^{\prime}\left(\mathbf{x}^{\prime}\right)=\left(\mathbf{A}^{\mathrm{T}}\right)^{-1} \mathbf{E}(\mathbf{x}), \mathbf{H}^{\prime}\left(\mathbf{x}^{\prime}\right)=\left(\mathbf{A}^{\mathrm{T}}\right)^{-1} \mathbf{H}(\mathbf{x}),
$$

and the new parameters in the transformed space are

$$
\boldsymbol{\varepsilon}^{\prime}\left(\mathbf{x}^{\prime}\right)=\mathbf{A} \boldsymbol{\varepsilon}(\mathbf{x}) \mathbf{A}^{\mathrm{T}} / \operatorname{det}(\mathbf{A}), \boldsymbol{\mu}^{\prime}\left(\mathbf{x}^{\prime}\right)=\mathbf{A} \boldsymbol{\mu}(\mathbf{x}) \mathbf{A}^{\mathrm{T}} / \operatorname{det}(\mathbf{A}),
$$

where $\mathbf{A}$ is the Jacobian matrix with components $A_{i j}=\partial x_{i}^{\prime} / \partial x_{j}$.

The most important information in the above analysis is that every solution of the Maxwell equations (either a propagating beam of light in free space or decaying evanescent waves in metals) in the original space ( $\mathbf{E}$ and $\mathbf{H}$ in Eq. (1)) is mapped to a corresponding solution in the transformed space ( $\mathbf{E}^{\prime}$ and $\mathbf{H}^{\prime}$ in Eq.(2)). We now have a recipe to "glue" the light rays to the coordinate system, as long as we transform the material parameters correspondingly according to Eq. 
(4). This is illustrated pictorially in Fig. 2 in two dimensions, in which a beam is bended after transformation, while from the coordinate mapping point of view, it is simply trying to follow those coordinates that corresponds to its original path in the original space. The parameters of the transformed space are obtained in Eq. (4). And the electromagnetic fields in the two spaces are related by Eq. (3).

x space

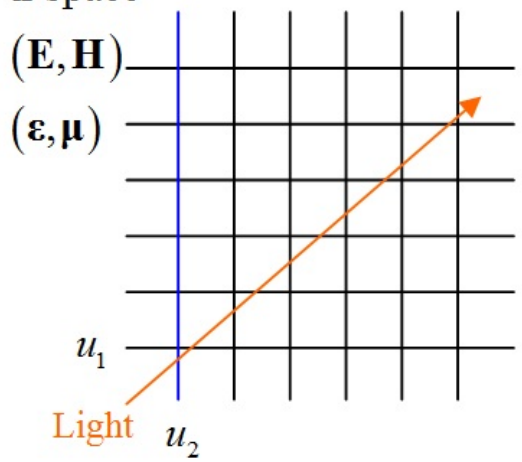

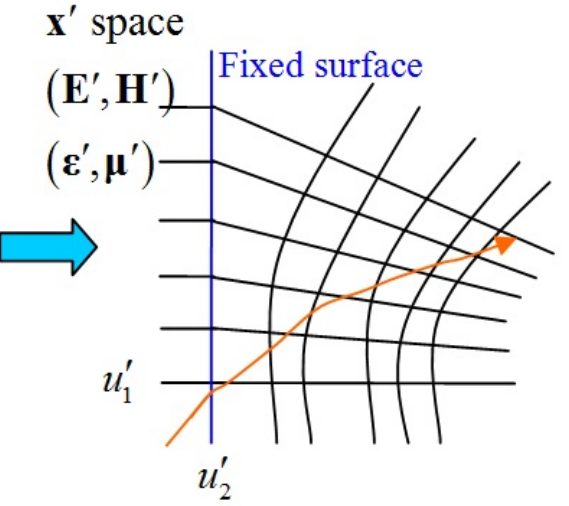

Fig. 2. The left panel shows a ray of light propagating in free space. The right panel shows the ray propagating in transformed space. The theory of transformation optics establishes a mapping of the trajectory of light between the original space and the transformed spaces, and the material parameters that is needed to perform the coordinate transformation.

We note that the Eqs.(1)-(4) do not depend on the choice of reference coordinates. In Fig. 2, we may use cylindrical coordinates instead of Cartesian coordinates, but as long as the mapping is fixed, the physical nature of original and new solutions would not be changed. For instance, if a surface (or a curve in two dimensions) is fixed (the blue line in Fig. 2) during the transformation, then we can choose to use the reference coordinate systems $\left(u_{1}, u_{2}\right)$ and $\left(u_{1}^{\prime}, u_{2}^{\prime}\right)$ before and after transformation (shown in Fig. 2), in which the fixed surface can be described as $u_{1}=u_{1}^{\prime}=c$. On the fixed surface, the Jacobian matrix elements $A_{i j}=\partial u_{i}^{\prime} / \partial u_{j}$ have $\partial u_{1}^{\prime} / \partial u_{2}=0$ and $\partial u_{2}^{\prime} / \partial u_{2}=1$, since each point on $u_{1}=c$ is mapped to the same point on $u_{1}^{\prime}=c$. Substituting into Eq. (3), we find that $E_{2}^{\prime}=E_{2}, H_{2}^{\prime}=H_{2}$, indicating that the tangential field components on the fixed surface are unchanged after transformation. This is an important point that is useful in many transformation optics devices as it assures that there is no reflection on the fixed surface boundary. 


\section{Complementary media}

As a simple example, we consider the coordinate transformation $x^{\prime}=-x$ (for $-d<x<0$ ) and $y^{\prime}=y, z^{\prime}=z$. This transformation corresponds to a "folding" of space [10, 11]. The Jacobian matrix can be written as $\mathbf{A}=\left(\begin{array}{ccc}-1 & 0 & 0 \\ 0 & 1 & 0 \\ 0 & 0 & 1\end{array}\right)$. From Eq.(4), the parameters of transformed space are obtained as $\boldsymbol{\varepsilon}^{\prime}\left(x^{\prime}, y^{\prime}, z^{\prime}\right)=-\boldsymbol{\varepsilon}(x, y, z), \boldsymbol{\mu}^{\prime}\left(x^{\prime}, y^{\prime}, z^{\prime}\right)=-\boldsymbol{\mu}(x, y, z)$. From Eq. (3), we obtain the following interesting relations: $\left(\begin{array}{c}E_{x}^{\prime}\left(x^{\prime}, y^{\prime}, z^{\prime}\right) \\ E_{y}^{\prime}\left(x^{\prime}, y^{\prime}, z^{\prime}\right) \\ E_{z}^{\prime}\left(x^{\prime}, y^{\prime}, z^{\prime}\right)\end{array}\right)=\left(\begin{array}{c}-E_{x}(x, y, z) \\ E_{y}(x, y, z) \\ E_{z}(x, y, z)\end{array}\right),\left(\begin{array}{c}H_{x}^{\prime}\left(x^{\prime}, y^{\prime}, z^{\prime}\right) \\ H_{y}^{\prime}\left(x^{\prime}, y^{\prime}, z^{\prime}\right) \\ H_{z}^{\prime}\left(x^{\prime}, y^{\prime}, z^{\prime}\right)\end{array}\right)=\left(\begin{array}{c}-H_{x}(x, y, z) \\ H_{y}(x, y, z) \\ H_{z}(x, y, z)\end{array}\right)$.

If the original space is free space, i.e. $\varepsilon=1, \mu=1$, then the transformed space is a slab with material parameters $\varepsilon=-1, \mu=-1$, as shown in Fig. 3(a). Such a slab is the "perfect lens", which is capable of not only achieving negative refraction [1], but also achieving a perfect image beyond the different limit, as was pointed out by Pendry [12].

If the original space is not free space, but is inhomogenous or contains objects, then the transformed space is slab composed of mirror images of the objects with negative parameters as those in the original space, as shown in Fig. 3(b). Such a slab called the "complementary media" $[13,14]$ of the original space. The notion of complementary media was actually introduced before transformation optics, but the unusual behavior of such media can be explained neatly using the language of transformation optics. Interestingly, although the original slab and its complementary media slab are inhomogeneous, they are still capable of achieving good imaging effect as illustrated in Fig. 3(c), where we show that a point source on the left surface of the original slab gives a point image on the right surface of the complementary media slab even though there are "objects" inside the slabs that can potentially lead to scattering. Actually, the complementary media slab is capable of copying arbitrary electromagnetic field values as well as their momentum on the left surface of the original slab to its right surface. To demonstrate this, we show in Fig. 3(d) a Gaussian beam incident on the left surface of the original slab and it tunnels to the right side of the complementary media slab and continues to propagate in its original direction. 

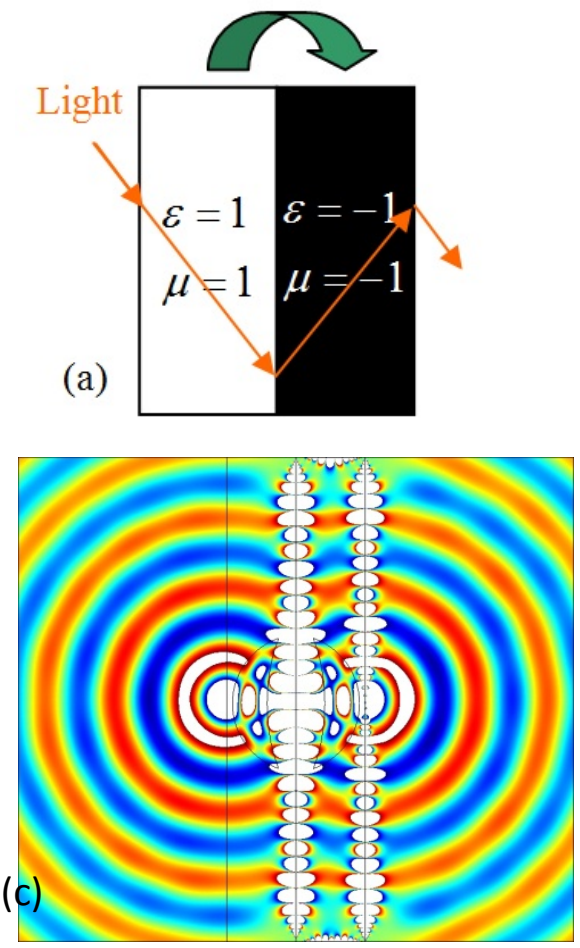
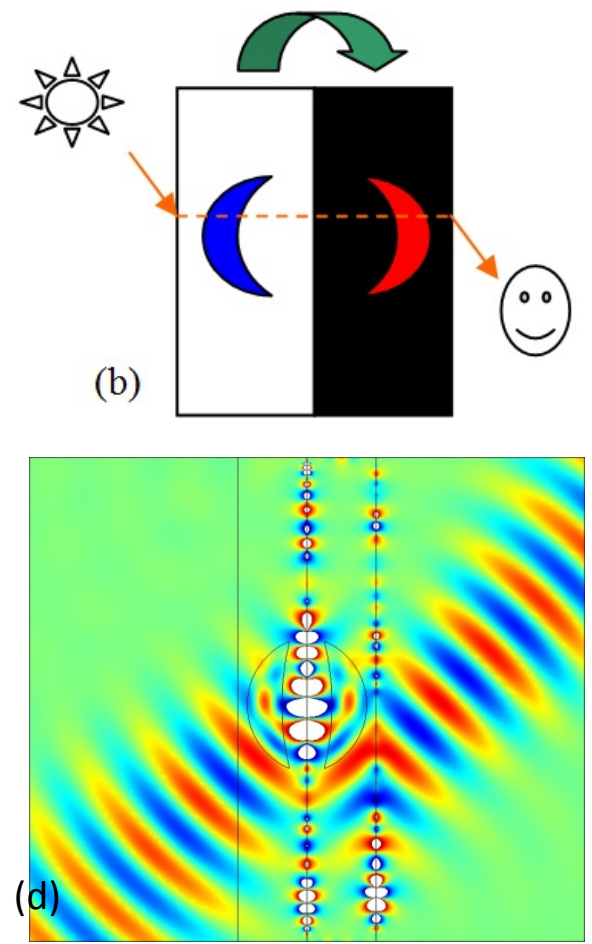

Fig. 3. (a) The operation of a perfect lens (a flat slab with $\varepsilon=\mu=-1$ ) can be explained using the concept of complementary media. The $\mathrm{n}=-1$ perfect lens slab optically cancels the space of a slab of air of the same thickness, effectively moving the source closer to the observer and hence enabling higher resolution. (b) An inhomogeneous slab (shown pictorially as a blue crescent in a white background) can be optically canceled by a complementary media slab shown pictorially as a red crescent in a black background. Wave propagates as if some space has disappeared. The effect of passing a wave through the complementary media is numerically demonstrated in (c) for a point source and in (d) for a Gaussian beam. In both cases, we note that the wave propagates as if the complementary media pair removes some space.

The above exotic behaviors of complementary media can be explained physically by space operations. From the point of view of transformation optics, complementary media slabs are formed by the operation of folding space. During the folding, the folding line (surface) is always fixed. According to our previous analysis, the tangential field components on the fixed surface are unchanged; therefore the boundary conditions between the complementary media slab and the original slab are always satisfied by the two solutions that are mapped to each other in the original and transformed spaces. Thus, the electromagnetic fields on the other side of original slab are also mapped to the other side of the complementary media slab. This leads to an interesting and intriguing consequence: the complementary media slab serves the function of cancelling the original slab of space. The canceled space can be simply removed from the system when we consider wave propagation and waves would go through the canceled 
space as if it did not exist at all. This can be used to explain the operation of a perfect lens. A perfect lens (a slab of $\varepsilon=\mu=-1$ ) of thickness $d$ is capable of optically cancelling spaces on either or both sides. The total thickness of the canceled slab spaces is also $d$. The observer is hence moved a distance $2 d$ closer to the light sources and as a result, the resolution is increased. By using the space cancellation concept, it is straightforward to understand the results shown in Figs. 3(c) and (d). The space cancellation function is so powerful that a lot of illusion tricks can emerge, as will be shown later. However, we should emphasize that space cancellation (and therefore complementary media) alone does not mean invisibility. To achieve invisibility, we need to make a certain domain behave optically as if it has $\varepsilon=\mu=1$. Light will have phase accumulation when it travels through an invisible domain. On the other hand, a complementary medium cancels space and wave travels through it without phase change. A beam of oblique incidence will also be shifted as it passes through a complementary medium. What a complementary medium can do is that it removes the identity of any object inside. For example, independent of the size, shape and composition of the embedded objects shown in the Fig. 3(c) and (d), a pair of complementary media will give exactly the same scattered wave. The outside observer can tell that some space is missing, but the identity of the objects inside the folded space is not detectable. We will see later that if we add some additional "restoring media" to the complementary media to compensate for the missing phase, i.e. restore a region of illusion free space, we can achieve invisibility.

\section{Invisibility cloaks}

In the pioneering works of transformation optics, the concept of invisibility cloaks has been proposed to illustrate the power of the method $[8,9]$. Such invisibility cloaks are sometimes referred to as Harry Potter's cloak as the invisibility cloaks have the "magical" effect of making objects or people invisible from all observation angles. But we should note here that up to now the invisibility cloaks can only operate in a narrow frequency range due to the limit of metamaterial dispersions. Moreover, the cloaked object is also "blind", i.e. it cannot see the outside world.

The design of invisibility cloaks are based on space operations using transformation optics. As shown in Fig. 4(a), light in free space travels in straight lines, as long as gravity is not considered. In order to achieve the effect of cloaking, one natural way is to bend the light such that it goes around a domain (encircled by the red curve) and exits from the other side with the original momentum as if it passes through a piece of free space, as shown in Fig. 4(b). The shell that bends the light is the invisibility cloak. It can be created by expanding a dot (red dot in Fig. 4(a)) in free space within a circle of free space (dashed curve in Fig. 4(a)) using transformation optics. As a result, the original space within the 
circle are squeezed into a shell with a hidden space that light does not go into, as shown in Fig. 4(c). On the other hand, the outer circle (blue curve in Fig. 4(c)) is a fixed surface on which the boundary conditions are automatically satisfied. This indicates that there would be no reflection or refraction under any incident waves for this device. Invisibility is therefore achieved with a hidden space inside which we can hide an object from being observed.

We note that similar coordinate transforms have been applied to the conductivity equation by Greenleaf, Lassas and Uhlmann. [15]. They proved that an object enclosed with a shell of anisotropic conductivities can escape detection by electrical impedance tomography and the mathematical transformation ("blow up" transform) employed is the same as the transformation used in obtaining electromagnetic invisibility cloaks.

In Fig. 4(d), we show a two-dimensional example for cylindrical invisibility cloak. In this case, it is more convenient to use cylindrical coordinates and the Jacobian matrix can be written as:

$$
\mathbf{A}=\left(\begin{array}{ccc}
\frac{\partial r^{\prime}}{\partial r} & \frac{\partial r^{\prime}}{r \partial \theta} & 0 \\
\frac{r^{\prime} \partial \theta^{\prime}}{\partial r} & \frac{r^{\prime} \partial \theta^{\prime}}{r \partial \theta} & 0 \\
0 & 0 & 1
\end{array}\right),
$$

in which $\left(r^{\prime}, \theta^{\prime}\right)$ denotes the space coordinate in the cloak, while $(r, \theta)$ denotes the coordinate in the original circular space. Here we take the simple "blow up" mapping of $r^{\prime}=(b-a) \frac{r}{b}+a$ and $\theta^{\prime}=\theta$, in which $a$ and $b$ denote the inner and outer radius of the invisibility cloak. The corresponding parameters for invisibility cloaks are obtained from Eq. (4) as $\varepsilon_{r}^{\prime}=\mu_{r}^{\prime}=\frac{b-a}{b} \frac{r}{r^{\prime}}$, $\varepsilon_{\theta}^{\prime}=\mu_{\theta}^{\prime}=\frac{b}{b-a} \frac{r^{\prime}}{r}$ and $\varepsilon_{z}^{\prime}=\mu_{z}^{\prime}=\frac{b}{b-a} \frac{r}{r^{\prime}}$. Here, $\left(\varepsilon_{z}^{\prime}, \mu_{r}^{\prime}, \mu_{\theta}^{\prime}\right)$ corresponds to TE polarization (electric field in $z$ direction) and $\left(\varepsilon_{r}^{\prime}, \varepsilon_{\theta}^{\prime}, \mu_{z}^{\prime}\right)$ corresponds to TM polarization (magnetic field in $z$ direction). In Fig. 4(d), we have used an incident plane wave with TE polarization from the left. The invisibility cloak has $a=0.5 \lambda_{0}$ and $b=1.4 \lambda_{0}$. It is clearly seen that such a cloak indeed does not scatter incident waves and has thus achieved invisibility. 

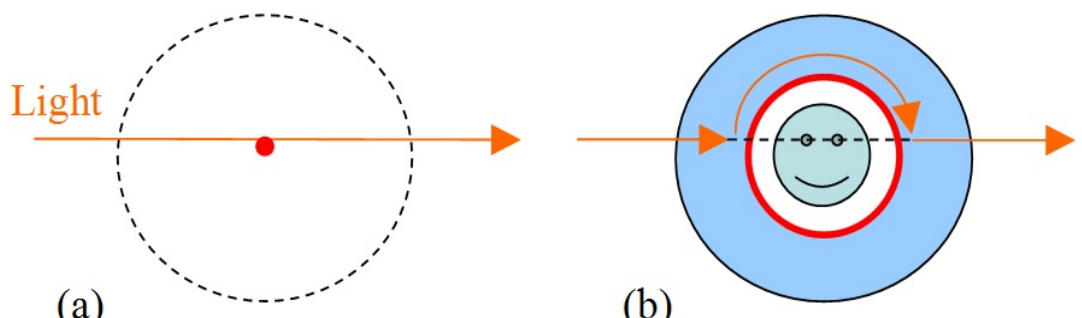

(b)
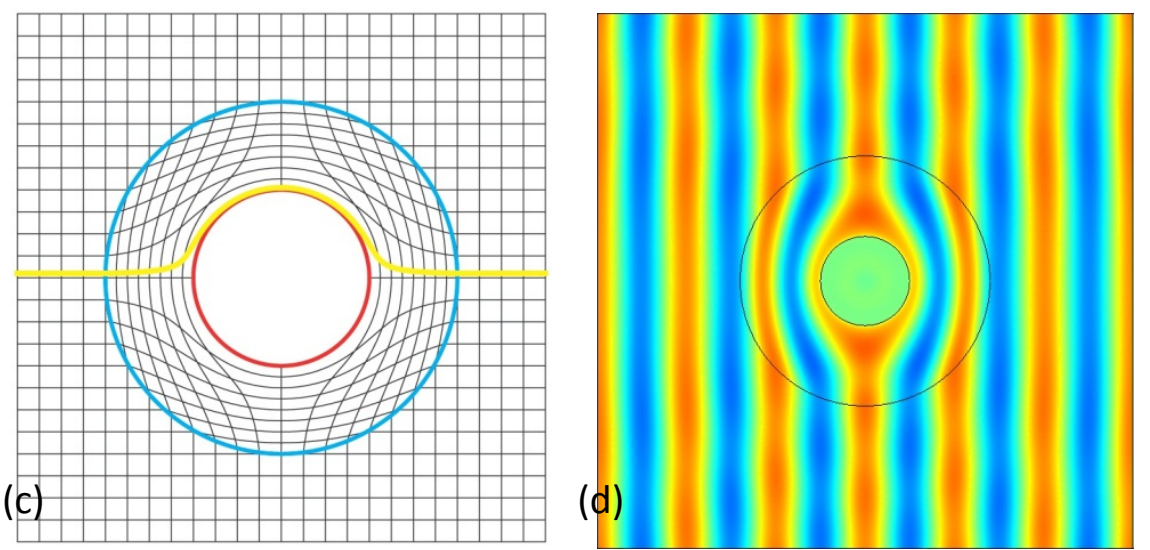

Fig. 4. (a) Light passing through free space. The dotted line represent the virtual boundary of a circle. (b) Light passing through a circular invisibility cloak obtained by expanding the red dot in free space into the circle marked by the solid red line. (c) The coordinate transformation corresponding to invisibility cloak introduced by Pendry and co-workers. (d) Numerical demonstration of cloaking effect. Waves go around the cloaked region. Any object placed inside the cloaked region will not cause scattering.

Realizing such a cloaking method is by no means straightforward and the difficulty lies in finding suitable materials for the perfect invisibility cloak, which requires some components of the permittivity and permeability tensors to go to zero or infinity. As a result, reduced parameters have been used in the first experiment by D. Schurig et al. [16], in which the effective refractive index, i.e. $\sqrt{\varepsilon_{z} \mu_{r}}$ and $\sqrt{\varepsilon_{z} \mu_{\theta}}$ are maintained as the original parameters, while the impedance matching is ignored. Plane wave pattern has proved the cloaking effect.

The invisibility cloak is capable of achieving invisibility at all observation angles. However, it is usually very demanding on metamaterials and difficult to realize. Actually, if only observation angles in the reflection geometry in half space are considered, the requirement on metamaterials can be greatly reduced. This is because that in reflection geometry the hidden space can be obtained by expanding from a surface instead of from a point. Such a cloak is called a carpet cloak, as shown in Fig. 5(a). In Fig. 5(b), we show the numerical demonstration of 
a carpet cloak under a Gaussian beam incident from top left. It is seen that the field pattern is as if the Gaussian beam is reflected back from a flat surface. Good cloaking effect is achieved. The original carpet cloak [17] employs quasiconformal mapping, which reduces the anisotropy to a minimum. If anisotropy is not an issue, the transformation mapping is actually more straightforward $[18,19]$. Here, we illustrate the operation of the carpet cloak with the set up shown in Fig.5(b) The cloak is made of three parts, i.e. two triangular parts with $\varepsilon_{z}^{\prime}=\mu_{x x}^{\prime}=2, \mu_{y y}^{\prime}=5$ and $\mu_{x y}^{\prime}= \pm 3$, formed by the coordinate transformation of $y^{\prime} \pm 6 x=1 / 2 \cdot(y \pm 6 x)$ and $x^{\prime}=x$, and one rectangular part with $\varepsilon_{z}^{\prime}=\mu_{x x}^{\prime}=2$ and $\mu_{y y}^{\prime}=0.5$, formed by the coordinate transformation of $y^{\prime}-0.6=1 / 2 \cdot(y-0.6)$ and $x^{\prime}=x$. Obviously, there are no singular parameters in the case of carpet cloaks. Therefore, carpet cloaks are much easier to realize in experiments. In fact, most of recent experiments on cloaking have been performed in such reflection geometry.

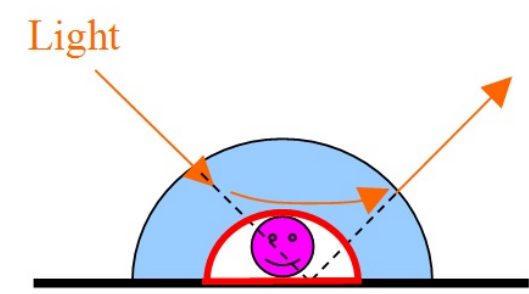

(a)

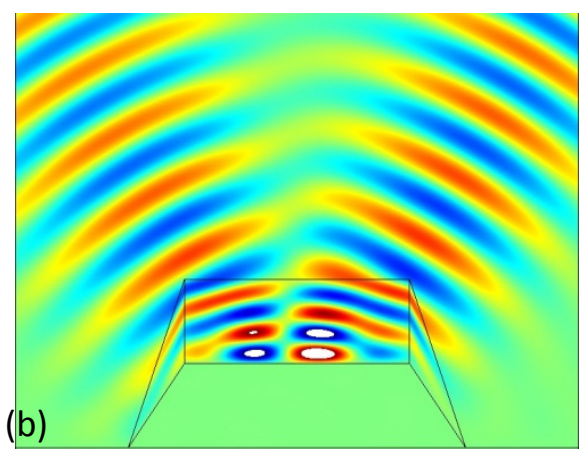

Fig. 5. (a) Schematic picture demonstrating the operation of a carpet cloak. (b) Numerical demonstration of the cloaking effect of a carpet cloak under a Gaussian beam incident from top left and getting reflected back.

Since light does not enter into the hidden space of the cloak obtained by "blow up" transforms, anything inside it is cloaked. However, this isolation also prevents objects hidden inside the cloak to "see" the outside world by light. In the following, we will show that there is actually a way to see the outside world while maintaining invisibility by using the notion of complementary media and transformation optics.

\section{Cloaking at a distance}

In the previous cloaking scheme of invisibility cloaks, a transformation optics device that has the same function as a piece of free space is created. The key 
principle behind is bending light around a domain using anisotropic materials, similar to the mirage effect. There are other cloaking schemes using transformation optics. In the following, we introduce the concept of "cloaking at a distance" [20], which makes objects invisible not by covering them with a metamaterial coating, but by placing a passive invisibility device at a distance to the objects, as shown pictorially in Fig. 6(a). Such a cloaking scheme uses the notion of complementary media to optically cancel some real space containing the object to be hidden and thereby remove its identity, and an illusionary free space is created using a restoring medium.

The mechanism of cloaking at a distance is shown pictorially in Fig. 6(b), in which a crescent-shaped object with material parameters $\left(\boldsymbol{\varepsilon}_{o}, \boldsymbol{\mu}_{o}\right)$ (blue colored) is made invisible by putting an invisibility device beside it. The invisibility device is circular and composed of two parts. The first part is a complementary media layer (black colored) formed by folding a layer of space (within the dashed circle) containing the object inward. This folding operation results in a layer of parameters $\left(\boldsymbol{\varepsilon}^{\prime}, \boldsymbol{\mu}^{\prime}\right)$, but embedded with a mirror object (an “anti-object”) with parameters $\left(\boldsymbol{\varepsilon}_{o}^{\prime}, \boldsymbol{\mu}_{o}^{\prime}\right)$ (red). As discussed before, the function of this complementary media layer may be regarded as cancelling the original space and mapping the field on the dashed circle to the inner surface of the complementary media layer, as indicated by the orange dashed lines. In such a way, the propagating light is transferred to propagate inside the core media (green). When the light ray touches the complementary media layer the second time, it will be transferred again to the dashed circle and propagate in free space again. Now, the key step to make the system identical to a circle of free space is to choose the core media as one of high refractive index formed conceptually by compressing the circle of free space into the core. In this way, the optical path experienced by light ray inside the core media will be exactly the same as that by light ray propagating crossing the dashed circle in free space, as shown by the green dashed line in Fig. 6(b). Therefore, any observers outside the dashed circle cannot see any scattered light from the object and the invisibility device, i.e. the system is invisible.

In short, the real space containing the object is cancelled optically by the complementary media, and the core media restores an illusionary free space to achieve the invisibility effect. Thus, the core media is called restoring media.

We now numerically demonstrate the phenomenon of cloaking at a distance in two dimensions. First, in Fig. 6(c), we show the scattering effect of a dielectric object of $\varepsilon_{o}=2$ under an incident plane wave of TE polarization from the left side. In Fig. 6(d), we place a corresponding invisibility device beside the object.

By using the linear mapping $r^{\prime}=(b-a) \frac{c-r}{c-b}+a$ and $\theta^{\prime}=\theta$ in Eq. (5), in which $a, b$, and $c$ are the inner radius, outer radius and the radius of the dashed circle, respectively, the complementary media layer material parameters can be 
obtained $\quad$ as $\quad \varepsilon_{r}^{\prime}=\mu_{r}^{\prime}=-\frac{b-a}{c-b} \frac{r}{r^{\prime}} \quad, \quad \varepsilon_{\theta}^{\prime}=\mu_{\theta}^{\prime}=-\frac{c-b}{b-a} \frac{r^{\prime}}{r} \quad$ and $\varepsilon_{z}^{\prime}=\mu_{z}^{\prime}=-\frac{c-b}{b-a} \frac{r}{r^{\prime}}$ for the region outside the mirror object. Inside the region of the "anti-object", the permeability is the same as the region outside, while the permittivity is changed correspondingly into $\varepsilon_{r}^{\prime}=-\frac{b-a}{c-b} \frac{r}{r^{\prime}} \varepsilon_{o}$, $\varepsilon_{\theta}^{\prime}=-\frac{c-b}{b-a} \frac{r^{\prime}}{r} \varepsilon_{o}$ and $\varepsilon_{z}^{\prime}=-\frac{c-b}{b-a} \frac{r}{r^{\prime}} \varepsilon_{o}$. In Fig. 6(d), we have chosen $a=0.5 \lambda_{0}, b=\lambda_{0}, c=2 \lambda_{0}$. The plane wave pattern indicates that the whole system has no scattering. Cloaking effect is evident.

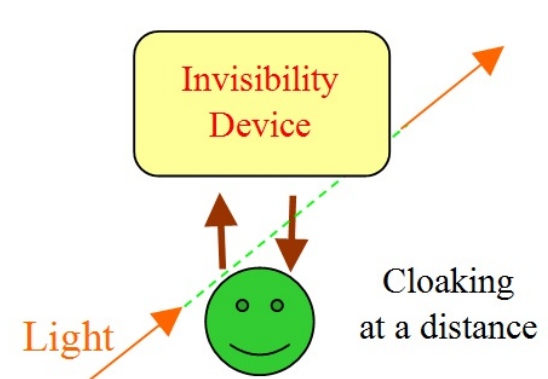

(a)

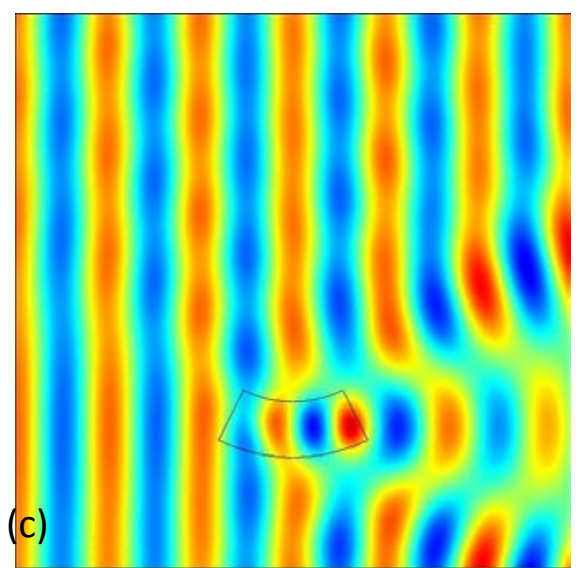

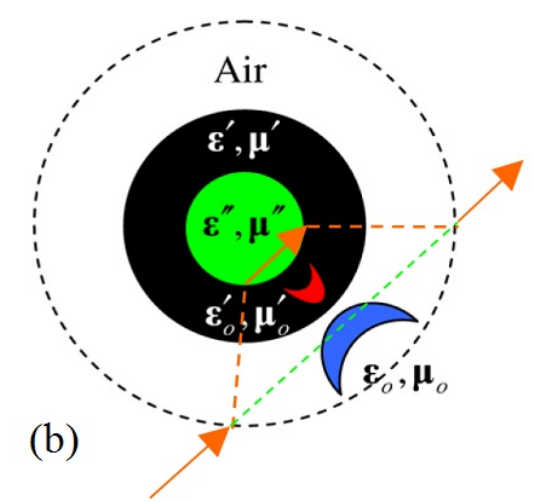

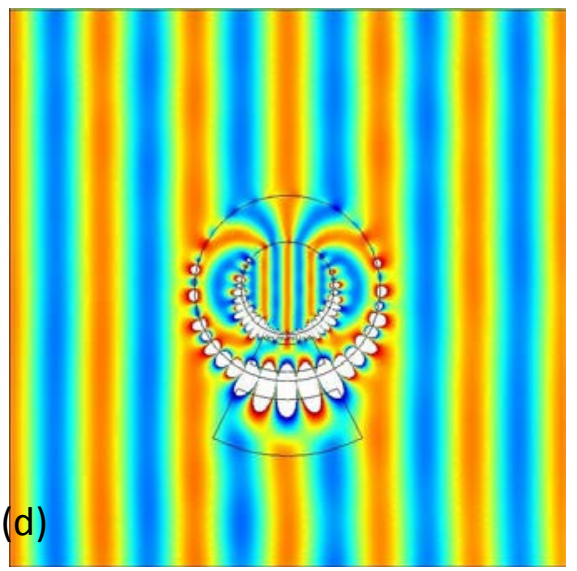


Fig. 6. (a) Schematic picture illustrating the idea of cloaking at a distance. (b) A picture illustrating the mechanism of cloaking at a distance. We want to make the object (blue moon) invisible without enclosing it. We cancel the space containing object (blue moon) by a shell of complementary media (shown pictorially as black shell with red moon. We restore the canceled space with an illusionary free space by restoring media (green core). For ordinary objects with positive refractive indices, the complementary media shell is required to have negative refractive indices. The restoring media (green core) typically has high refractive index. (c) Numerical demonstration of the scattering of an object in free space. (d) Numerical demonstration of cloaking the object at a distance with an invisibility device. We note that the plane wave front is now restored and the object is not detectable at the designed frequency. However, wave enters the object and the object is not "blind".

In this scheme of cloaking at a distance, we note that the incident waves interact with the object directly, thus the object can still "see" the outside world, while being "cloaked" at a distance by the invisibility device at the same time. This is different from the original invisibility cloak scheme in which the cloaked object has no contact with the incident wave.

Like the original invisibility cloaks proposed in 2006, the mechanism of cloaking at a distance is also a result of space manipulations, and as a result, the cloak should work irrespective of the property of the incident light as well as the observation angle. This is illustrated in Fig. 7. In Figs. 7(a)-(c), we demonstrate the effect of cloaking under various incident waves, including a plane wave incident from another direction (Fig. 7(a)), a point source (Fig. 7(b)) and a Gaussian beam (Fig. 7(c)). The cloak works as expected for different incident waves. However, unlike invisibility cloaks, cloaking at a distance works only when the invisibility device and the object are placed at a designed distance. In Fig. 7(d), we show the scattered wave pattern of the invisibility device by itself when the same Gaussian beam as that in Fig. 7(c) impinges on the cloaking device. Obviously scattering occurs. The remote invisibility device itself is not invisible as a standalone object, but it is invisible when it is placed at the predesigned position next to the object to be cloaked.
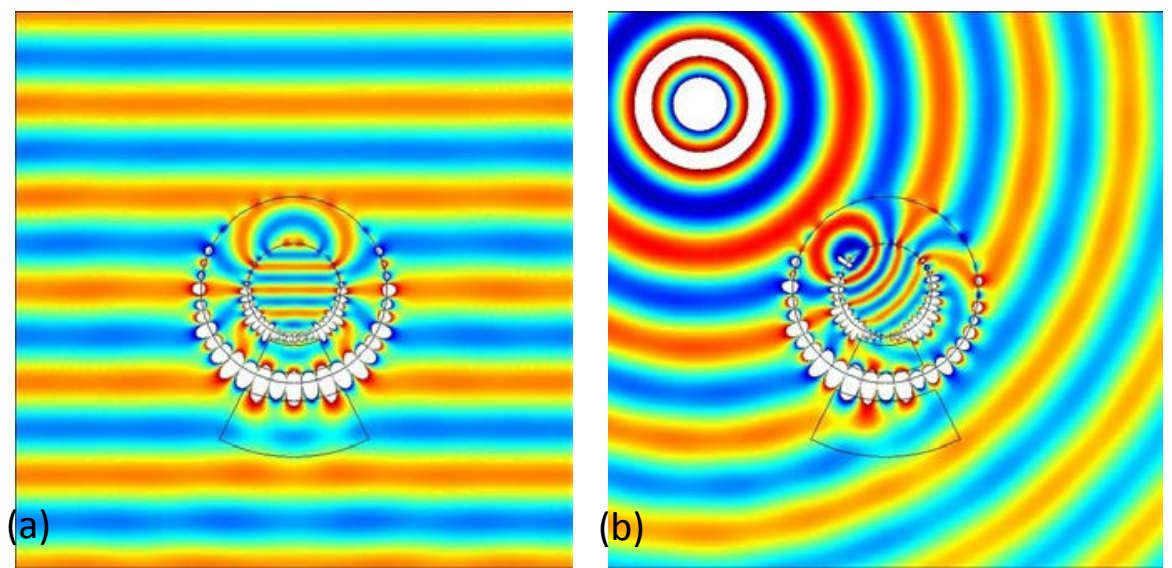
(c)
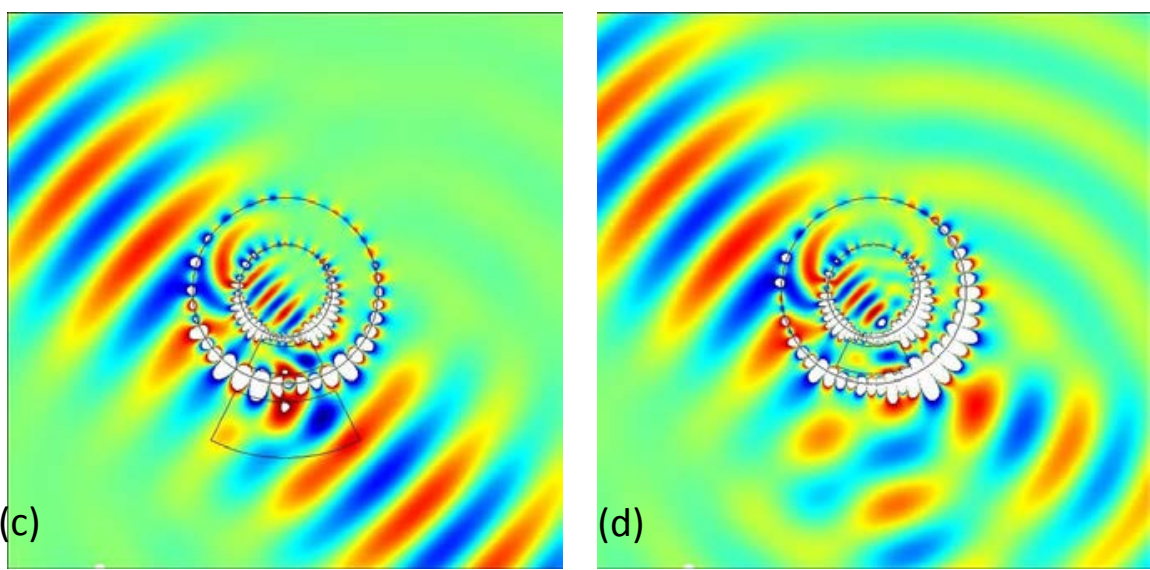

Fig. 7. Numerical demonstrations of cloaking a distance. Panels (a) to (c) shows the performance of the cloak under different forms of illumination: (a) under a plane wave incident from top; (b) a point source at up left corner; (c) a Gaussian beam incident from top left. These demonstrations show that remote cloak works irrespective of the direction and the nature of the incident wave. (d) The scattering of the invisibility device itself under the Gaussian beam.

From the viewpoint of wave interference, cloaking at a distance has demonstrated an example of achieving far-field destructive interference of all scattered waves by employing passive devices with strong multiple scattering interactions with the object to be "cloaked". This is an effect more subtle than the original invisibility cloak. However, the price to pay is the complexity of the metamaterial. We need to employ negative index metamaterial with low-loss in the complementary media layer. The metamaterial should be able to produce strong surface waves in the boundaries, as seen in Fig. 6(d) and Figs. 7(a)-(c). As low loss double negative metamaterials are always difficult to make, particularly in high frequencies, the material issue here is at least as challenging as that in invisibility cloaks that operates by light bending. The requirement may be somewhat less stringent in the sense that there is no extreme parameters (zero or infinity) in the complementary media. Nevertheless, transmission line samples have been fabricated with negative parameters at microwave frequencies and the effect of cloaking at a distance has been demonstrated experimentally. We note in passing that the cloaking effect here is not the same the cloaking effect discovered by Milton and co-workers [21, 22] who demonstrated that a small dipolar object can be made undetectable as a result of "anomalous resonance" when it is placed close enough to a perfect lens with a negative refractive index. The anomalous resonance effect can cloak an object only if certain conditions are satisfied. The object must be very small and must be placed close to the negative index slab. There is no "anti-object" or "restoring medium". In our approach, the underlying mechanism is the space cancellation effect of complementary media. There is no limit to the size of the object to be cloaked and there is in principle no limit to the distance between the device and the cloak if absorption is small. 


\section{Making part of an object invisible}

A significant advantage of applying folding action in space manipulation is the ability to make part of an object invisible [23]. In Fig. 8, we show the numerical demonstration of such an operation. In Fig. 8(a), we show the scattering pattern when plane wave impinges on a thin dielectric layer of $\varepsilon_{o}=2$. In Fig. 8(b), we use an invisibility device that is purposely designed to cloak just the upper part of a thick dielectric layer, leaving the lower part untouched. The "anti-object" is designed so that it only cancels part of the object. It is seen that the scattering pattern is the same as that of a lower thin dielectric layer, i.e. the upper part is made invisible while leaving the lower part unaffected. As the scattering patterns of configurations in Figs. 8(a) and 8(b) are the same to an outside observer; the observer will "see" an thin object instead of a thicker object.
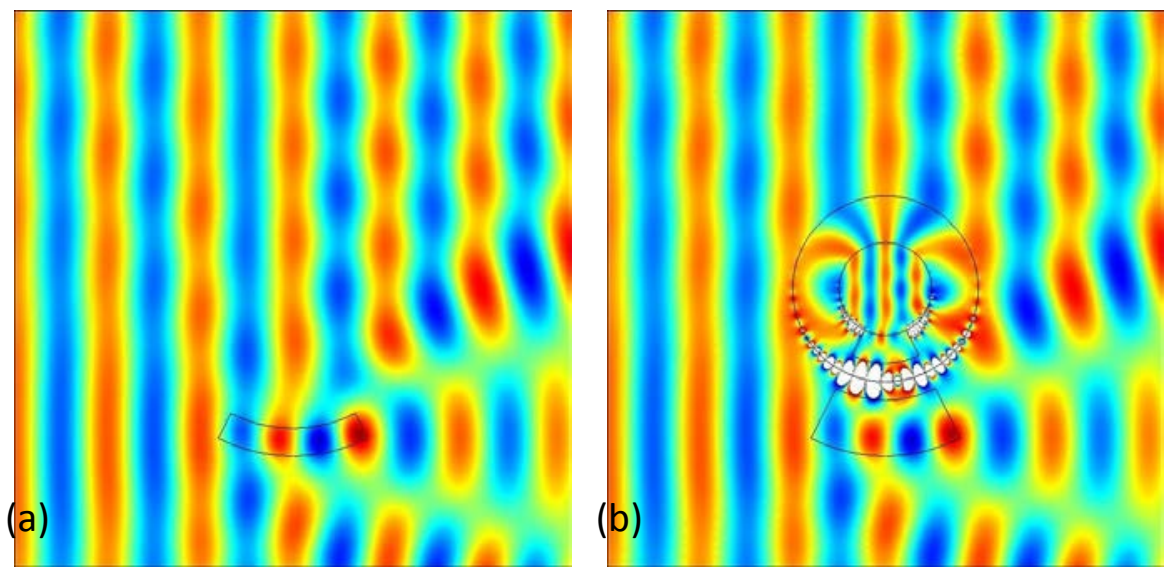

Fig. 8. (a) Scattering of plane wave by a dielectric object. (b) The anti-object is designed purposely to make the upper part of a thick dielectric layer invisible, while leaving the lower part unaffected. As a consequence of this partial cloaking effect, the scattering of the object in (b) becomes the same as the scattering due to the object in (a) even though the object in (b) is bigger in size. A far field observer will believe that they are seeing the object shown in panel (a).

Such a special effect is a derivative of cloaking at a distance and may lead to many interesting applications. For example, a wall of negative permittivity would certainly block electromagnetic waves, as most metals do. Information is thus blocked by the wall and we cannot see through the wall. In order to see information on the other side, one can of course drill a hole on the wall. However, we can obtain information non-invasively using the concept of remote cloaking [24]. Using transformation optics, we can make part of the wall invisible, which means that a virtual hole is opened on the wall through which light can tunnel into the outside as if there is a real hole. 
In Fig. 9(a), we show that electromagnetic waves radiated from a point source is blocked by a wall of $\varepsilon_{o}=-1$. In Fig. 9(b), we show a transformation scheme that serves to open a virtual hole on such a wall. The invisibility device is also made of a complementary media part (black) and a restoring media part (green). Their combining function is to first optically cancel one part of the wall (bounded by black dashed lines), and then restore the canceled space with illusion of free space. When a beam of light (orange arrow) hits on left side of the wall, it tunnels to the other side. This is the property of complementary media. Then, it is guided to emit with the original momentum and proper position by the restoring media. The numerical demonstration of attaching an invisibility device on the outside of the wall is shown in Fig. 9(c). We see that electromagnetic waves can now propagate to the right side of the wall. In Fig. 9(d), we show the simulation of electromagnetic waves propagating through a real hole drilled on a wall. It is seen that the radiation pattern in the far field is almost the same as that in Fig. 9(c). We note that the restoring medium (green colored part) is not needed if we just need light to leak out from the wall, but it is needed if we want the outgoing field to be the same as if a real hole was drilled in the wall.

In Fig. 9(c), the geometry of the invisibility device is not cylindrical. The complementary media part is obtained by folding the canceled trapezoidal part of wall to the left black part, i.e. $x^{\prime}=-x / 2$, which results in an homogeneous medium of $\varepsilon_{z}^{\prime}=2, \mu_{x}^{\prime}=-0.5$ and $\mu_{y}^{\prime}=-2$. The restoring media part is formed by compressing an illusionary free space bounded by the black dashed lines into the region of the restoring media (green). Here, we take the simplest way of compressing two triangular parts and one rectangular part in the $x$ direction, as shown in Fig. 9(c). The upper and lower triangular parts are of $\varepsilon_{z}^{\prime}=4$, $\mu_{x x}^{\prime}=9.25, \mu_{y y}^{\prime}=4$ and $\mu_{x y}^{\prime}=\mp 6$, formed by the coordinate transformation of $x^{\prime} \pm 2(y \mp 0.5)=1 / 4 \cdot(x \pm 2(y \mp 0.5))$ and $y^{\prime}=y$, respectively. The middle rectangular part is of $\varepsilon_{z}^{\prime}=4, \mu_{x}^{\prime}=0.25$ and $\mu_{y}^{\prime}=4$, formed by the coordinate transformation of $x^{\prime}-0.2=1 / 4 \cdot(x-0.2)$. 

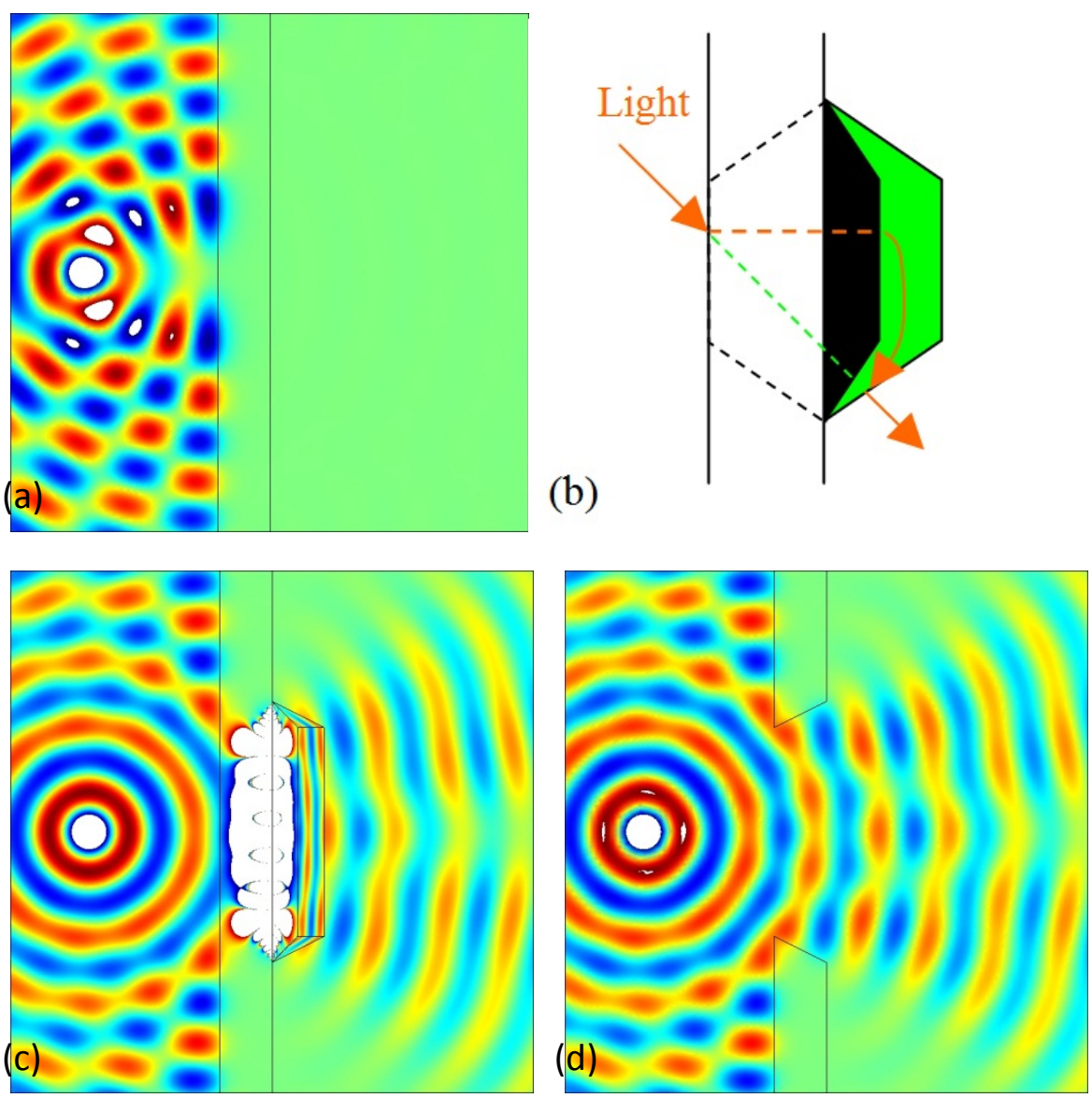

Fig. 9. (a) Numerical simulation of a point source placed on one side of a wall blocking electromagnetic waves. (b) Schematic design of a device that can make a virtual hole on the wall so that light can tunnel through the wall via the complementary (black) and restoring (green) media. (c) Numerical demonstration of waves propagating through the virtual hole to the other side. The source is the same as that in panel (a). (d) Numerical demonstration of waves propagating through a real hole.

By using similar designs, such invisibility devices also enable interesting applications like turning a box into free space so that we can see what is hidden inside the box. In addition to numerical demonstrations, we also have a mathematical proof for the remote cloaking and illusion optics effect. The interested reader may refer to the supplementary material of Ref. [24]. 


\section{Other cloaking schemes}

In addition to the invisibility cloak and cloaking at a distance, there are some other approaches to achieve invisibility. The brief description below is not intended to be comprehensive, but just to show that transformation optics can provide many solutions to the same problem.

In one approach, an object is coated with metamaterial layers, such that the combined system can be regarded as illusionary free space. This method requires interaction between the object and the metamaterial layer. The simplest example is a homogeneous dielectric material of arbitrary shapes in two dimensions for TE polarization. As shown in Fig. 10(a), by covering the dielectric object (purple) with certain transformation media, it is possible to perfectly guide the light into the dielectric object and then out into free space at the other side. As a result, all the light travels as if passing through an illusion free space (dashed lines). In Fig. 10(b), we show the example of cloaking a dielectric object by covering it with a transformation medium that guide light into the object. Such a transformation medium is called a "concentrator" [25]. Recently, a variant of this cloaking scheme have been proposed using only single negative materials [26]. However, such an approach works only for making certain types of objects (that can be transformed into illusionary free space by transformation optics) invisible.

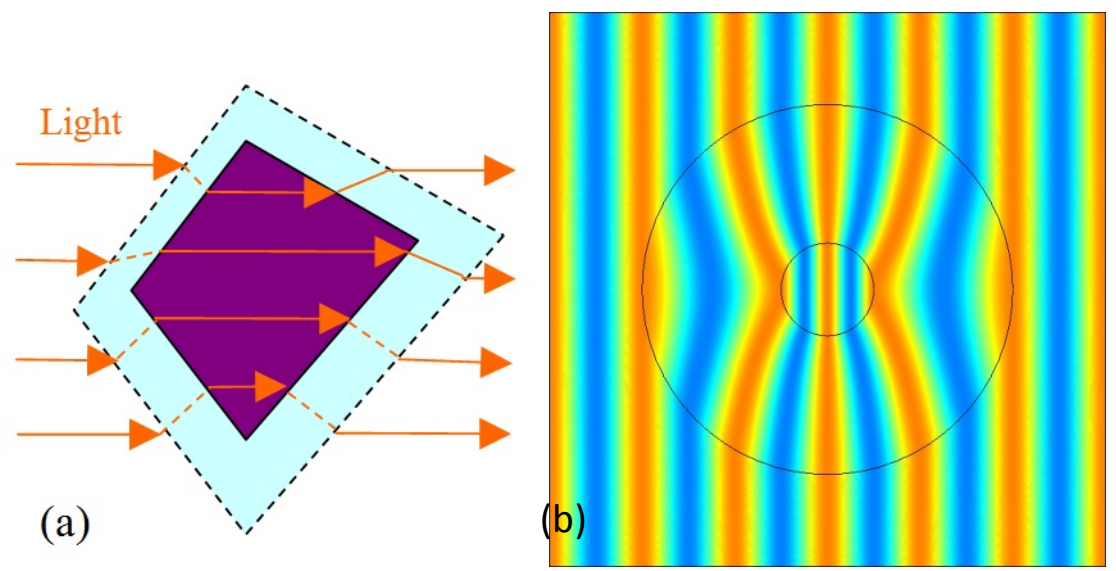

Fig. 10. (a) Demonstration of guiding light into an object by covering it with a transformation medium. (b) Numerical demonstration of a concentrator, which is an invisible device with a high refractive index core.

A schematic graph of the coordinate mapping in cylindrical radial coordinate is shown in Fig. 11 to demonstrate the different cases of invisibility cloaks, cloaking at a distance and the wave concentrator. In Fig. 11(a), we show the coordinate mapping of invisibility cloaks, in which a cloak between $R_{1}$ and $R_{2}$ are formed by 
this mapping, which can be used to cloak any objects with $R_{1}$ (shown as an apple in Fig. 11(a)). In Fig. 11(b), we show the coordinate mapping of cloaking at a distance, in which any objects between $R_{2}$ and $R_{3}$ (shown as an apple) can be made invisible by the "anti-object" (a red mirror image of the apple) embedded within the complementary media layer (between $R_{1}$ and $R_{2}$ ) as well as the restoring medium (within $R_{1}$ ). In Fig. 11(c), we show the mapping of concentrator, which is invisible too.

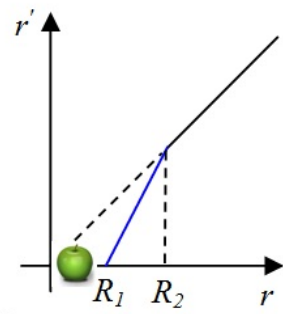

(a)

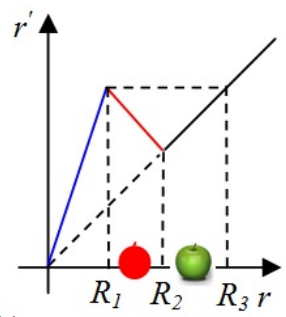

(b)

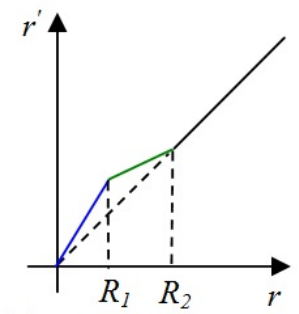

(c)

Fig. 11. Coordinate mappings in radial cylindrical coordinates: (a) Radial "blow up" mapping that achieves invisibility cloaks. (b) The mapping that can achieve of cloaking at a distance. The red color part gives materials with negative refractive index. (c) The mapping corresponding to a wave concentrator.

There are other types of cloaking that also employs cancellation. For example, A. Alu et al. have proposed to use a plasmonic coating to "cloak" a small object. We can regard the negative permittivity layer serves to cancel the first order scattering of the dielectric core [27]. However, as the mechanisms are not based on transformation optics and space manipulation and we will not discuss it in details here.

\section{From invisibility to illusion optics}

We have previously shown that invisibility can be achieved by manipulation of optical spaces, i.e. turning the real space with objects into an illusionary free space without object. Indeed, both the light-bending invisibility cloak approach and the cloaking at a distance approach can be considered as creating an illusionary free space using transformation optics. With this knowledge, one simple question is: what if the illusionary space is not free space, but contains some illusion objects?

It turns out that it is feasible to create arbitrary stereoscopic passive illusions. For example, we can in principle make an apple look like a banana. Here, by passive illusions, we mean that the illusion of a banana is not generated by active devices such as TVs or projectors, but that the far field optical property of the 
apple is indeed turned into that of the banana. Whatever light is incident upon the system, the scattered light will be exactly the same as that from a banana instead of an apple, therefore making an observer to believe that a banana is there instead of an apple. This amazing effect is achieved by replacing the real space with an apple into an illusion space with a banana, as shown in Fig. 12, and is therefore denoted as Illusion optics [24].

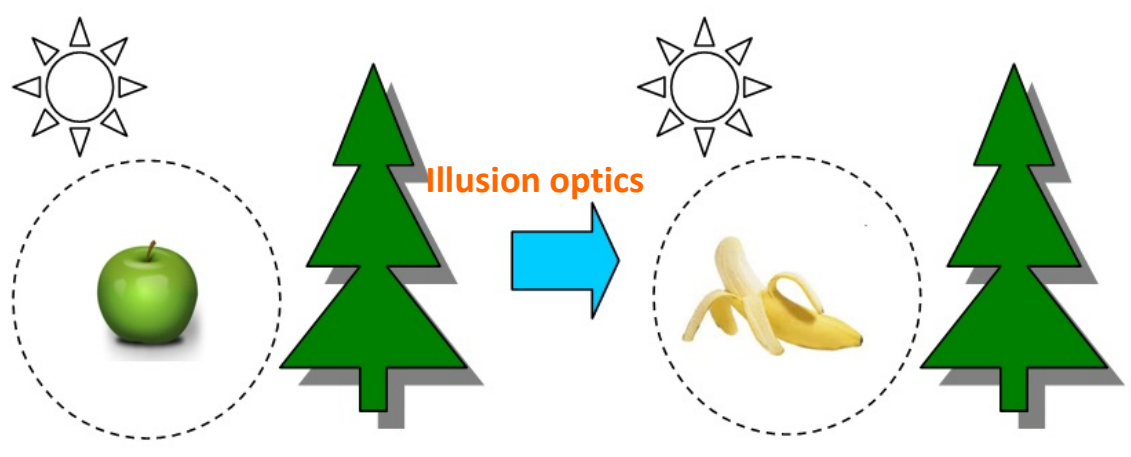

Fig. 12. Schematic graph of optically replacing a region of real space containing one object (an apple) into that of an illusion space containing an illusionary object (an banana), such that any observer will see a banana instead of an apple. Everything outside the region is optically unchanged.

As an example, we consider making a spoon look exactly like a cup outside a certain boundary. In Fig. 13(a), we show the computed scattering pattern of a spoon of $\varepsilon_{o}=2$ when plane wave is incident from the left. In Fig. 13(b), we demonstrate the schematic graph of using a passive illusion device to transform the optical response of the dielectric spoon into that of a cup of $\varepsilon_{o}=-1$. The illusion device is similar in composition to the invisibility device in Fig. 9(b). It is composed of two parts, a complementary media part that cancels the optical space containing the spoon, and a restoring media part that refills in the illusionary optical space with the illusion cup. As a result, any light incident upon this region would propagate as if there is only a cup inside. The difference of illusion device with the invisibility device is that there are illusionary objects in the illusionary space instead of pure free space. We note that the negative refractive index component in this device serves two purposes. As a component of the complementary media, it "cancels" the object. It also serves as a lens that forms the image of the cup. 

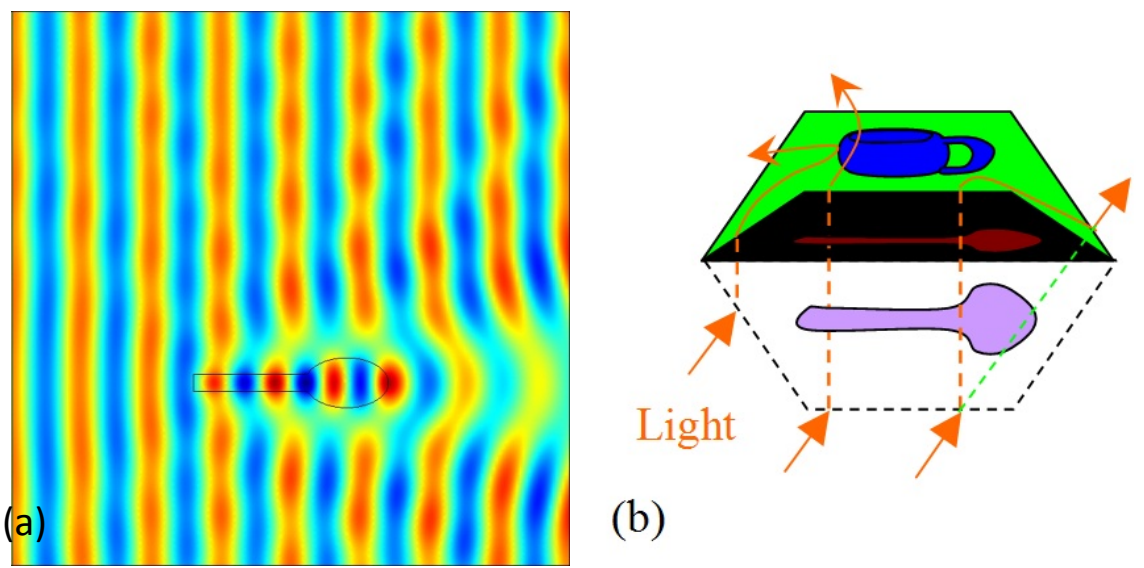

(b)
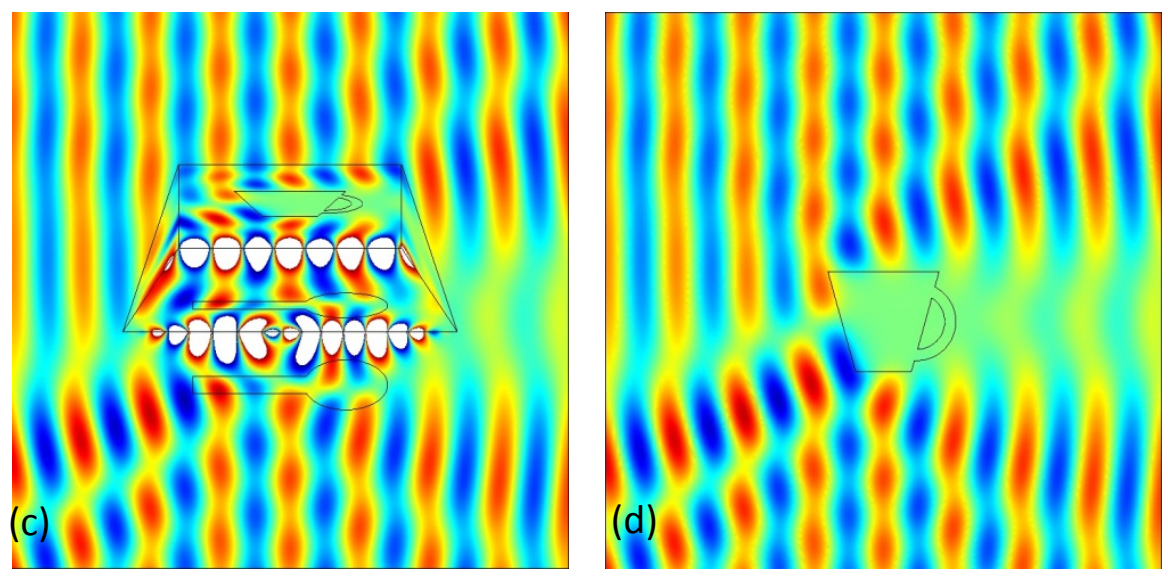

Fig. 13. (a) The scattering of plane waves by a spoon-like object. (b) Schematic graph of using an illusion device to transform the optical response of a spoon into that of a cup, an example of illusion optics. (c) Numerical demonstration of optically transforming the spoon into the cup. Comparing with the numerical simulation in panel (d), which shows the scattering pattern of a cup, we see that the total scattering of the device and the spoon becomes the same as that of a standalone cup. The outside observer will see a cup instead of a spoon.

The material parameters of the illusion device that we used in Fig. 13(c) are similar to those of the invisibility device in Fig. 9(b), with the exchange between $x$ and $y$ directions and additional insertion of an "anti-object" in the complementary media part as well as an illusion object in the restoring media part. As shown in Fig. 13(c), the scattering pattern of whole system is changed by the illusion device. By comparing it with the scattering pattern of a single cup in free space, which is shown in Fig. 13(d), it can be seen that the two patterns are almost exactly the same in the far field region. This indicates that the optical scattering of a spoon is indeed changed into that of a cup by the passive illusion device. Any 
observer will see a cup instead of a spoon at a distance. The illusion effect is thus demonstrated numerically.

Similar to invisibility devices, there are also more than one approaches to achieve illusions. We have demonstrated creating illusion using the cancelling and restoring approach, similar to cloaking at a distance. In fact, the invisibility cloak can be modified to create illusions as well [28]. As shown in Fig. 14, by expanding a point (red dot) in a space with a crescent-shaped object, it is possible to obtain an illusion cloak that has exactly the same function as the original crescent-shaped object in free space. In the illusion cloak, any object can be hidden without being seen. Therefore, it is also capable of achieving stereoscopic illusions. Since the extension is straightforward, we will not give numerical examples for this case.

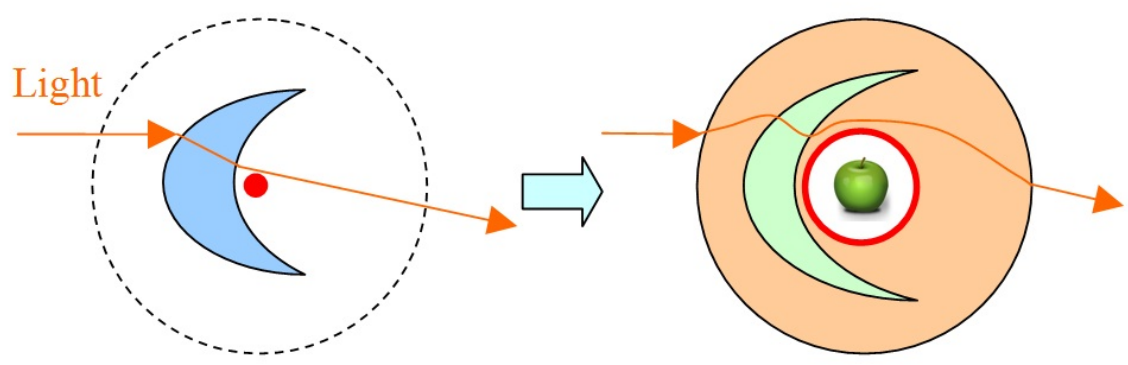

Fig. 14. Schematic graph of an illusion cloak that creates illusions. Any object placed inside the red circle will look like the crescent colored in blue.

By using illusion optics, it is possible to change the optical response of a region of space almost at will and there are many plausible applications. In the following, we will introduce two examples: the super-scatterer which has a scattering cross section that is much bigger than the geometric cross section, and the superabsorber that can absorb light beyond its physical boundaries. There are many other applications that we cannot cover here such as a rotator that creates illusionary rotating effect, shifter that creates illusionary shifting effects, the remote control of light in cavities, shrinked optical devices, and ground illusion devices that create illusions in the reflection geometry, etc. The interested readers may refer to the references.

\section{Super-scatterers}

One interesting example of illusion is the super-scattering effect [29]. The mechanism of super-scatterer is shown pictorially in Fig. 15(a). It is composed of a complementary media layer surrounding a PEC core (which repels waves here), such that any light incident on the virtual boundary of the device would tunnel to 
the surface of the PEC core and tunnel back, as if reflected at the virtual boundary. In other words, a super-scatterer creates an illusion a larger PEC. In Fig. 15(b), we demonstrate the scattering pattern of the PEC core in free space under an incident plane wave from the left. In Fig. 15(c), we show the scattering pattern of the super-scatterer, and in Fig. 15(d), the scattering pattern of an illusion PEC. It is immediately seen that the scattering pattern in the far field are almost the same in Figs. 15(c) and (d). The illusion PEC effect is proved.

The parameters of the complementary media layer are the same as that in Fig. 6(d) except that there is no "anti-object" embedded inside.
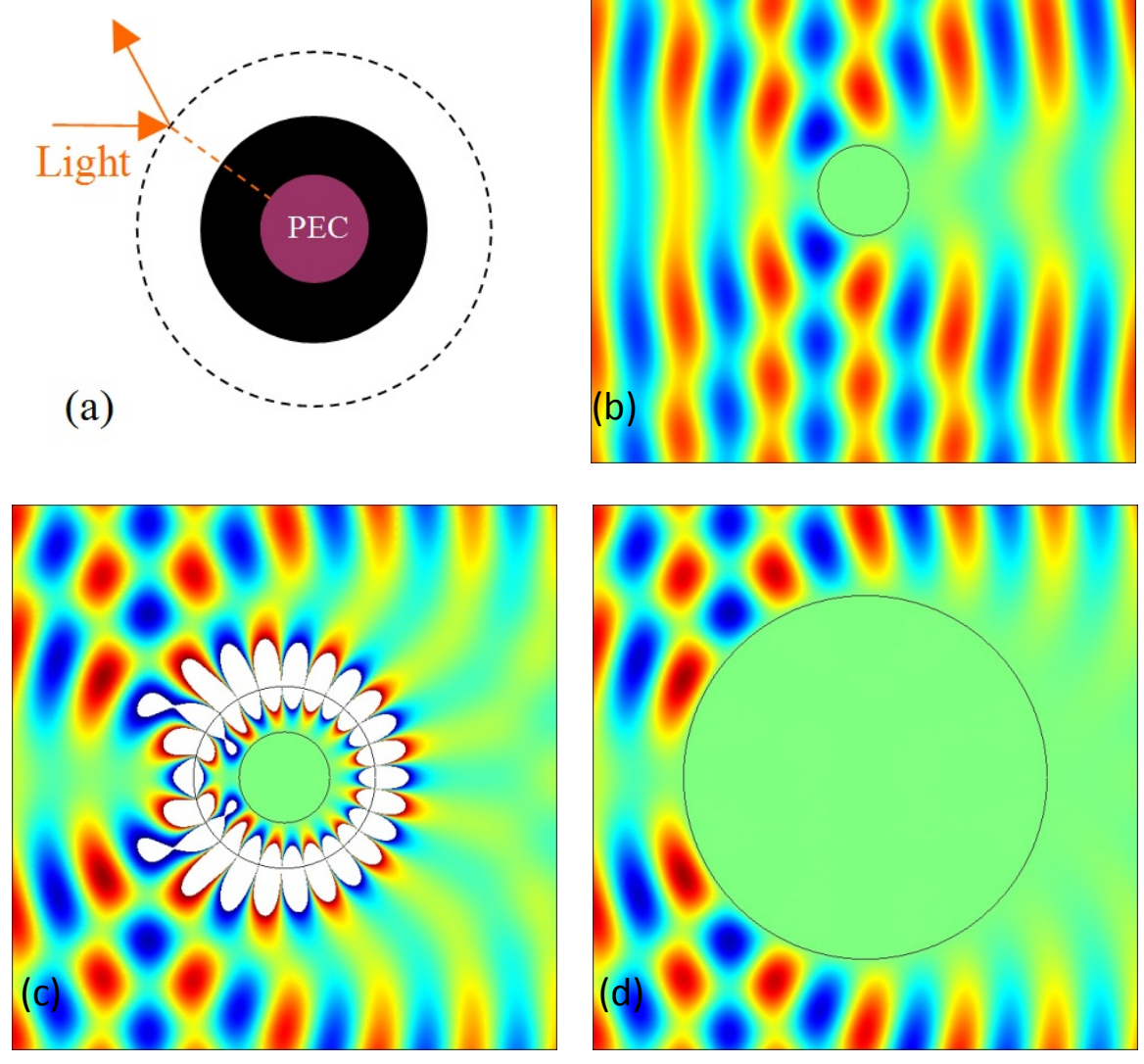

Fig. 15. (a) Schematic picture of a super-scatterer composed of a perfect electric conductor (PEC) and a complementary media layer. (b) The scattering pattern of the PEC. (c) The scattering pattern of the super-scatterer. (d) The scattering pattern of a illusionary larger PEC. 
Super-scatterer can also be achieved in Cartesian geometry. In Fig. 12(c), if we remove the original spoon object, as well as the mirror spoon in the complementary media part and the illusionary cup in the restoring media part, and then we change the whole restoring media part into some materials that reflect light, then the illusion device turns into a super-scatterer, i.e. a scatterer with a much larger scattering cross section than its physical size. A schematic graph of the mechanism of super-scatterer has been shown in Fig. 16(a). Here we consider TE waves and we use the perfect electric reflectors (PECs) as the reflecting material. According to the function of complementary media, a beam of light incident on the virtual boundary (dashed lines) will be tunneled to the other side and get reflected by PEC boundary, and then tunneled back to propagate in another direction, as if it is reflected at the virtual boundary. In the view of space manipulations, such an illusion device creates an illusionary PEC which is much larger than the true one.

Numerical demonstration of such a super-scatterer is shown in Fig. 16(b), in which the scattering pattern is very similar to the scattering pattern of a single larger PEC, as shown in Fig. 16(c). This proves the effect of the super-scatterer. The super-scatterer has an interesting application. It can be used to create a hidden portal. As shown in Fig. 16(d), a super-scatterer is embedded in a PEC wall. The electromagnetic waves will be reflected by an illusion of a continuous PEC wall, and therefore cannot transfer to the other side. However, there is a real gap in the wall in which physical objects or waves of other frequencies can go through [30].
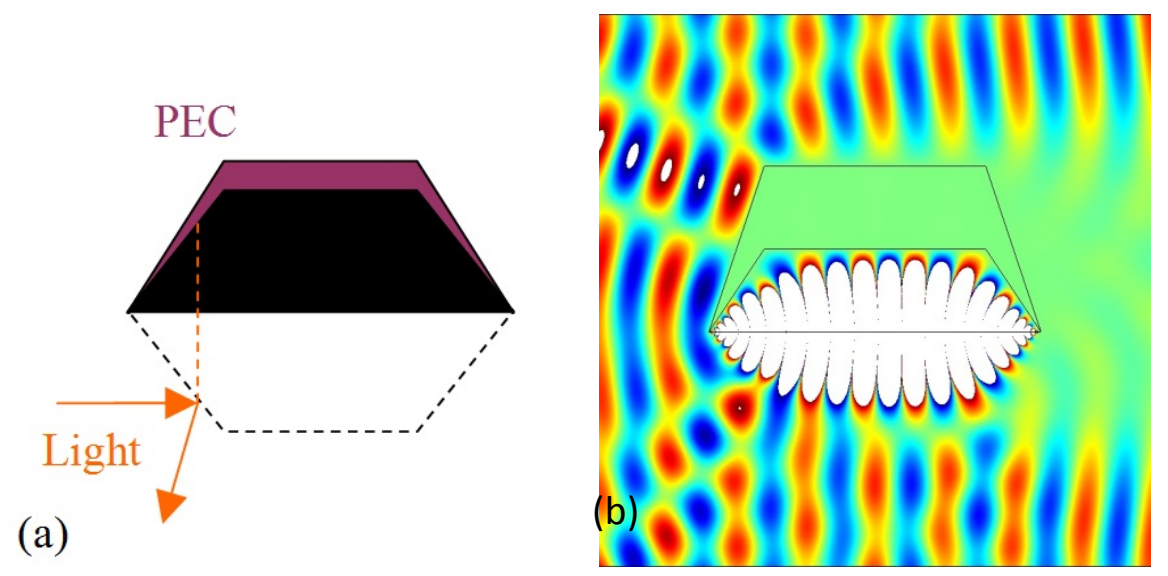

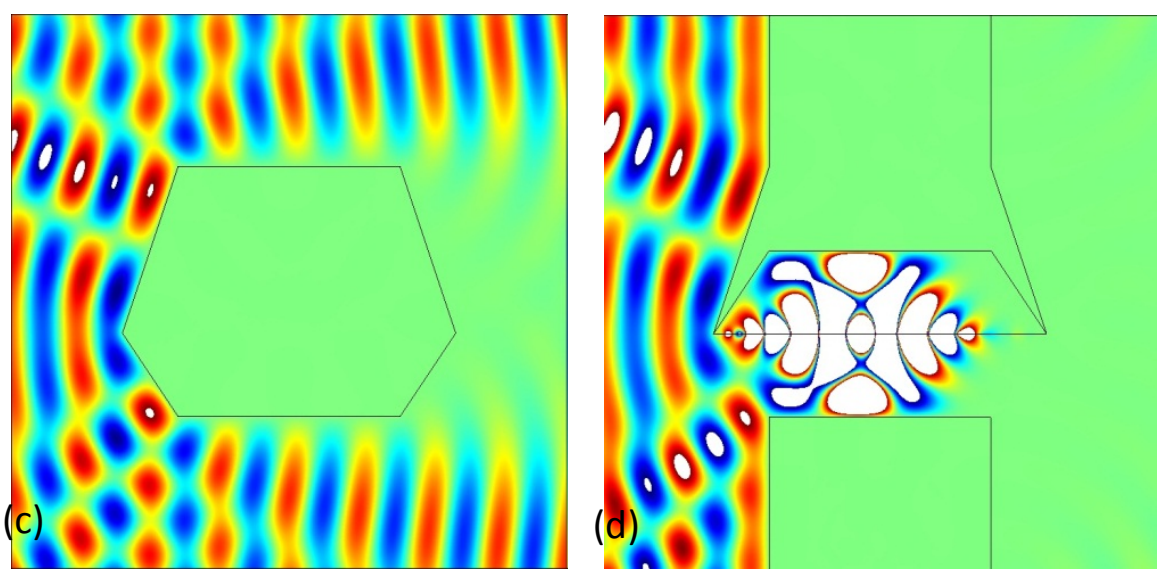

Fig. 16. (a) Schematic picture of a super-scatterer in the Cartesian geometry. (b) Scattering pattern of the super-scatterer. (c) Scattering pattern of an illusionary larger PEC. (d) Realization of a hidden portal with the super-scatterer.

\section{Super-absorbers}

Another illusion effect is the super-absorber effect, where one enlarges an absorptive core by using illusion optics [31]. In most known situations, the absorption cross section can only be slightly bigger than the geometric cross section except when we have small particles at resonance. While small particles can absorb more light than a blackbody of the same size, the absorption cross section cannot increase without bound for fixed particle volume. Here we shall show that using illusion optics, it is possible to build a super absorber who absorption cross section can increase significantly beyond the physical cross section at the working frequency, and the effect is not limited to small particles.
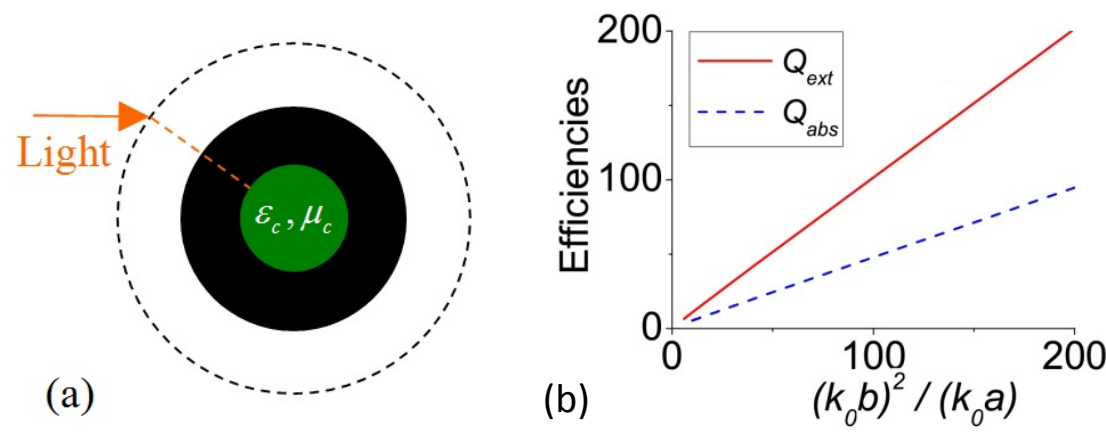
Fig. 17. (a) The geometry of the super absorber, which consists of a core of radius $a$ and a shell of radius $b$. The core has constitutive parameters of $\varepsilon_{c}$ and $\mu_{c}$, and the shell has constitutive parameters of $\varepsilon_{s}=-1$ and $\mu_{s}=-b^{4} / r^{4}$ at the working frequency. (b) Extinction and absorption efficiencies for the super absorber with $\varepsilon_{c}=(1+i) b^{4} / a^{4}, \mu_{c}=(1+i), k b=2$ and $a$ is varying.

For simplicity, we consider the problem in two dimensions. The problem can be generalized to three dimensions in a straightforward manner. The geometry of the super absorber, which consists of a core and a shell, is illustrated in Fig. 17(a). In this case, instead of using a linear mapping, we have used the mapping of $r=b^{2} / r^{\prime}$ for $a<r<b$, in which $a$ and $b$ are the core radius and the outer radius of the complementary media layer. The resulting complementary media parameters are $\varepsilon_{s}=-1$ and $\mu_{s}=-b^{4} / r^{4}$. Such a system would effectively behave as an illusionary cylinder of radius $r^{\prime}=b^{2} / a$ with a "diluted" version of the absorbing core parameters, i.e. $\varepsilon_{c}=(1+i)$ and $\mu_{c}=(1+i)$. We consider an illumination by transverse electric waves at normal incidence. Fig. 17(b) shows the extinction efficiency and absorption efficiencies for a super absorber. It is seen that one can maintain the super absorber at the same physical size (fixed $b$ ), while increase its efficiencies by decreasing $a$. When $a$ tends to zero, the absorbing power of the system would increase without bound. Of course, for $a$ too small, the constitutive parameters will become extreme.

\section{Other illusion ideas and Experiments}

There are many other interesting ideas of illusion optics. For example, it has been proposed to embed an "anti-object" in a ground of negative parameters, so as to cloak an object on the ground without using a carpet cloak [32]. The same approach is also capable of casting illusions in the reflection geometry. It has also been proposed to make illusion effects of rotating [33], shifting and reshaping [34, 35], changing materials [36], anti-cloaking [37], and optically remote controlling [38]. They can all be understood via the concept of illusion optics, i.e. the replacement of optical spaces.

It is challenging to realize various kinds of illusion effects at optical frequencies. This is due to the difficulty in realizing nanoscale metamaterials with suitable parameters. However, it is much easier to do experiments at microwave frequencies as the metamaterial fabrication is much easier at that length scale. Most of the experimental works are focusing on realizing carpet cloaks [18,19,39- 
43]; there also emerge a few experiments on invisibility cloaks [16], illusion optics $[44,45]$ and cloaking at a distance [46]. As the experimental technique improves with time, experimental demonstrations will be easier to carry out.

\section{Conclusions}

We have demonstrated that optical spaces can be manipulated by using transformation optics and metamaterials (see Refs. [47-50] for reviews). We can optically isolate or cancel a piece of space with objects inside and restore into a piece of illusionary space with arbitrary illusion objects. In such a way, the optical properties of the original object are changed into that of the illusion object outside a pre-specified boundary, creating a perfect illusion that cannot be distinguished from reality by observers in the far field. This is called illusion optics, first proposed in Ref. [24] and highlighted in Ref. [51]. In a netshell, real optical space can be replaced with an arbitrary illusion optical space, therefore enabling the creation of arbitrary stereoscopic optical illusions, including invisibility.

Potential application of illusion optics include various kinds of cloaking effects and illusion ideas such as invisibility cloaks, cloaking at a distance, superscatterers, super absorbers and many others. The idea of optical space replacement may be extended to more intriguing concepts such as cloaking with non-Euclidean spaces [52], optical black holes [53] and wormholes [54].

We have demonstrated some cases in two dimensions, but the principle works also for cases in three dimensions. Besides electromagnetic waves, it is also possible to create illusion for acoustic waves and other classical waves that are form-invariant under coordinate transformation (see e.g. [55-60]).

Acknowledgments: This work is supported by Hong Kong CRF grant HKUST2/CRF/11G. LY is supported by the State Key Program for Basic Research of China (No. 2012CB921501), National Natural Science Foundation of China (No. 11104196), Natural Science Foundation of Jiangsu Province (Grant No. BK2011277), Program for New Century Excellent Talents in University (NCET), and a Project Funded by the Priority Academic Program Development of Jiangsu Higher Education Institutions (PAPD).

References:

[1] Veselago V.G.: The electrodynamics of substances with simultaneously negative values of $\varepsilon$ and $\mu$, Sov. Phys. Usp. 10, 509 (1968).

[2] Pendry J.B., Holden A.J., Robbins D.J., Stewart W.J.: Magnetism from conductors and enhanced nonlinear phenomena, IEEE Trans. Microwave Theory Tech. 47, 2075 (1999).

[3] Shelby R.A., Smith D.R., Schultz S.: Experimental verification of a negative index of refraction, Science 292, 77 (2001).

[4] Smith D.R., Padilla W.J., Vier D.C., Nemat-Nasser S.C., Schultz S.: Composite Medium with Simultaneously Negative Permeability and Permittivity, Phys. Rev. Lett. 84, 4184 (2000) 
[5] Yao J., Liu Z.W., Liu Y.M., Wang Y. Sun G. Bartal G., Stacy A.M., Zhang X.:Optical negative refraction in bulk metamaterials of nanowires, Science 321, 930 (2008)

[6] Valentine J., Zhang S., Zentgraf T., Ulin-Avila E., Genov D.A., Bartal G., Zhang X.:Threedimensional optical metamaterial with a negative refractive index, Nature 455, 376 (2008)

[7] See, for example, Xiao S.M., Drachev V.P., Kildishev A.V., Ni X.J., Chettiar, U.K., Yuan H.K. Shalaev V.M.:Loss-free and active optical negative-index metamaterials, Nature 466, 735 (2010)

[8] Leonhardt U.: Optical conformal mapping, Science 312, 1777 (2006).

[9] Pendry J.B., Shurig D., Smith D.R.: Controlling electromagnetic fields, Science 312, 1780 (2006).

[10] Leonhardt U., Philbin T.G.: General relativity in electrical engineering, New J. Phys. 8, 247 (2006).

[11] Milton G.W., Nicorovici N.A.P., McPhedran R.C., Cherednichenko K., Jacob, Z.: Solutions in folded geometries, and associated cloaking due to anomalous resonance, New J. Phys. 10, 115021 (2008)

[12] Pendry J.B.: Negative refraction makes a perfect lens, Phys. Rev. Lett. 85, 3966 (2000).

[13] Pendry J.B., Ramakrishna S.A.: Near field lenses in two dimensions, J. Phys. Condens. Matter 14, 8463 (2002).

[14] Pendry J.B., Ramakrishna S.A.: Focusing light using negative refraction, J. Phys. Condens. Matter 15, 6345 (2002).

[15] Greenleaf A., Lassas M., Uhlmann G.: Anisotropic conductivities that cannot be detected by EIT, Physiol. Meas. 24, 413-419 (2003).

[16] Schurig D., Mock J.J., Justice B.J., Cummer S.A., Pendry J.B., Starr A.F, Smith D.R.: Metamaterial electromagnetic cloak at microwave frequencies, Science 314, 977 (2006).

[17] Li J., Pendry J.B., Hiding under the carpet: a new strategy for cloaking, Phys. Rev. Lett. 101, 203901 (2008).

[18] Zhang B.L., Luo Y., Liu X.G. , Barbastathis G.: Macroscopic Invisibility Cloak for Visible Light, Phys. Rev. Lett. 106, 033901 (2011)

[19] Chen X.Z., Luo Y., Zhang J.J., Jiang K., Pendry J.B., Zhang S.: Macroscopic invisibility cloaking of visible light, Nat. Comm. 2, 176 (2011)

[20] Lai Y., Chen H.Y., Zhang Z.Q., Chan C.T.: Complementary media invisibility cloak that cloaks objects at a distance outside the cloaking shell, Phys. Rev. Lett. 102, 093901 (2009).

[21] Milton G.W., Nicorovici N.A. P.: On the cloaking effects associated with anomalous localized resonance, Proc. R. Soc. A 462, 3027-3059 (2006).

[22] Dong J.W., Zheng H.H., Lai Y., Wang H.Z., Chan C.T.: Metamaterial slab as a lens, a cloak, or an intermediate, Phys. Rev. B 83, 115124 (2011).

[23] Lai Y., Ng J., Chen H.Y., Zhang Z.Q., Chan C.T.: Illusion Optics, Front. Phys. China, 2010, 5(3): 308-318 (2010).

[24] Lai Y., Ng J., Chen H.Y. Han D.Z., Xiao J.J., Zhang Z.Q., Chan C.T.: Illusion optics: the optical transformation of an object into another object, Phys. Rev. Lett. 102, 253902 (2009).

[25] Rahm M., Schurig D., Roberts D.A., Cummer S.A., Smith D.R., Pendry J.B.: Design of electromagnetic cloaks and concentrators using form-invariant coordinate transformations of Maxwell's equations, Photo. Nano. Funda. Appl. 6, 87-95 (2008).

[26] Zhu X.F., Liang B., Kan W.W., Zhou X.Y., Cheng J.C.: Acoustic Cloaking by a Superlens with Single-Negative Materials, Phys. Rev. Lett. 106, 014301 (2011).

[27] Alu A., Engheta N.: Achieving transparency with plasmonic and metamaterial coatings, Phys. Rev. E 72, 016623 (2005).

[28] Jiang W.X., Ma H.F., Cheng Q., Cui T.J.: Illusion media: Generating virtual objects using realizable metamaterials, Appl. Phys. Lett. 96, 121910 (2010).

[29] Yang T., Chen H.Y., Luo X.D., Ma H.R.: Superscatterer: Enhancement of scattering with complementary media, Opt. Express 16, 18545 (2008).

[30] Chen H.Y., Chan C.T., Liu S.Y., Lin Z.F.: A simple route to a tunable electromagnetic gateway, New J. Phys. 11, 083102 (2009). 
[31] Ng J., Chen H.Y., Chan C.T.: Metamaterial frequency-selective superabsorber, Opt. Lett. 34, 644 (2009).

[32] Wu K.D., Wang G.P.: Hiding objects and creating illusions above a carpet filter using a Fourier optics approach, Opt. Express 18, 19894 (2010).

[33] Chen H.Y., Chan C.T.: Transformation media that rotate electromagnetic fields, App. Phys. Lett. 90, 241105 (2007)

[34] Chen H.Y., Zhang X.H., Luo X.D., Ma H.R., Chan C.T.: Reshaping the perfect electrical conductor cylinder arbitrarily, New J. Phys. 10, 113016 (2008).

[35] Jiang W.X., Cui T.J.: Moving targets virtually via composite optical transformation, Opt. Express 18, 5161 (2010).

[36] Jiang W.X., Ma H.F., Cheng Q., Cui T.J.: Virtual conversion from metal object to dielectric object using metamaterials, Opt. Express 18, 11276 (2010).

[37] Chen H.Y., Luo X.D., Ma H.R., Chan C.T.: The anti-cloak, Opt. Express 16, 14603 (2008).

[38] Liang Z.X., Lin X.L., Jiang X.Y.: Remote control of light behavior by transformation optical devices, Opt. Express 18, 2049 (2010).

[39] Liu R., Ji C., Mock J.J., Chin J.Y., Cui T.J., Smith D.R.: Broadband ground-plane cloak, Science 323, 366 (2009).

[40] Valentine J., Li J., Zentgraf T., Bartal G., Zhang X.: An optical cloak made of dielectrics, Nat. Mater. 8, 568 (2009).

[41] Gabrielli L.H., Cardenas J., Poitras C.B., Lipson M.: Silicon nanostructure cloak operating at optical frequencies, Nat. Photon. 3, 461 (2009).

[42] Ergin T., Stenger N., Brenner P., Pendry J.B., Wegener M.: Three-dimensional invisibility cloak at optical wavelengths, Science 328, 337 (2010).

[43] Ma H.F., Cui T.J.: Three-dimensional broadband ground-plane cloak made of metamaterials, Nat. Comm. 1, 21 (2010).

[44] Li C., Meng X.K., Liu X., Li Fang, Fang G.Y., Chen H.Y., Chan C.T.: Experimental realization of a circuit-based broadband illusion-optics analogue, Phys. Rev. Lett. 105, 233906 (2010).

[45] Jiang W.X., Cui T.J.: Radar illusion via metamaterials, Phys. Rev. E 83, 026601 (2011).

[46] Li C., Liu X., Liu G.G., Li F., Fang G.Y.: Experimental demonstration of illusion optics with "external cloaking" effects, Appl. Phys. Lett. 84, 084104 (2011).

[47] Chen H.Y., Chan C.T., Sheng P., Transformation optics and metamaterials, Nat. Mater. 9, 387 (2010).

[48] Kundtz N.B., Smith D.R., Pendry J.B.: Electromagnetic design with transformation optics, Proc. IEEE 99, 1622 (2011).

[49] Wegener M., Linden S., Shaping optical space with metamaterials, Physics Today 63, 32 (2010)

[50] Liu Y.M., Zhang X.: Metamaterials: a frontier of science and technology, Chem. Soc. Rev. 40, $2494(2011)$

[51] Pendry J.B., All smoke and metamaterials, Nature 460, 579 (2009).

[52] Leonhardt U., Tyc T.: Broadband Invisibility by Non-Euclidean Cloaking, Science 323, 110 (2009).

[53] Genov D.A., Zhang S., Zhang X.: Mimicking celestial mechanics in metamaterials, Nat. Phys. 5, 687-692 (2009).

[54] Greenleaf A., Kurylev Y., Lassas M., Uhlmann G.: Electromagnetic Wormholes and Virtual Magnetic Monopoles from Metamaterials, Phys. Rev. Lett. 99, 183901 (2007).

[55] Milton G.W., Briane M., Willis J.R.: On cloaking for elasticity and physical equations with a transformation invariant form, New J. Phys. 8, 248 (2006).

[56] Chen H.Y., Chan C.T.: Acoustic cloaking and transformation acoustics, J. Phys. Conden. Phys. 43, 113001 (2010).

[57] Zhang S., Xia C.G., Fang N.: Broadband Acoustic Cloak for Ultrasound Waves, Phys. Rev. Lett. 106, 024301 (2011).

[58] Popa B.I., Zigoneanu L., Cummer S.A.: Experimental Acoustic Ground Cloak in Air, Phys. Rev. Lett. 106, 253901 (2011). 
[59] Farhat M., Guenneau S., Enoch S.: Ultrabroadband Elastic Cloaking in Thin Plates, Phys. Rev. Lett. 103, 024301 (2009).

[60] Stenger N., Wilhelm M., Wegener M.: Experiments on Elastic Cloaking in Thin Plates, Phys. Rev. Lett. 108, 014301 (2012). 